\title{
A MAC protocol for Underwater Sensors Networks
}

\author{
Rodrigo Santos ${ }^{1}$, Javier Orozco ${ }^{1}$, Sergio F. Ochoa ${ }^{2}$, Roc Messeguer ${ }^{3}$, Gabriel Eggly ${ }^{1}$ \\ 1 Dep. Electrical Engineering and Computers, IIIE, UNS-CONICET \\ 2 Computer Science Department, Universidad de Chile \\ ${ }^{3}$ Computer Science Department, Universidad Politecnica de Catalunya
}

\begin{abstract}
Underwater sensor networks are becoming an important field of research, because of its everyday increasing application scope. Examples of their application areas are environmental and pollution monitoring (mainly oil spills), oceanographic data collection, support for submarine geo-localization, ocean sampling and early tsunamis alert. It is well-known the challenge that represents to perform underwater communications provided that radio signals are useless in this medium and a wired solution is too expensive. Therefore, the sensors in these network transmit their information using acoustic signals that propagate well under water. This data transmission type bring an opportunity, but also several challenges to the implementation of these networks, e.g., in terms of energy consumption, data transmission and signal interference. Few proposals are available to deal with the problem in this particular application scenario, and these proposals does not address properly the transmission of underwater acoustic signals. In order to help advance the knowledge in the design and implementation of these networks, this paper proposes a MAC protocol for acoustic communications between the nodes based on a self-organized time division multiple access mechanism. The proposal is still preliminary and it has only been evaluated in the laboratory; however, it represents a highly promising behavior that make us expect interesting results in real-world scenarios.
\end{abstract}

Keywords: Acoustic Sensor Networks, Collaborative Systems, Opportunistic Routing

\section{Introduction}

The ocean covers 71 percent of the Earth's surface and contains 97 percent of the planet water, yet more than 95 percent of the underwater world remains unexplored. UNESCO states that over 90 percent of the earth habitable space is within the ocean, and near 80 percent of all life is under the ocean surface. There are quite dramatic projections stating that by the year 2100 more than half of the marine species may stand on the brink of extinction [1]. This shows only some of the several reasons that we have to deploy underwater technology that help us study several phenomena that affect our lives and the health of our planet.

In this scenario, the underwater sensor networks could provide valuable information that helps us address some of these challenges [2]. However, underwater radio communications are practically unfeasible, specially in the ocean because radio signals suffers a strong attenuation, limiting the effective communication to a few meters. Acoustic 
signals instead are capable of traveling for long distances, depending on the power used for the transmission and the physical characteristics of the medium. Therefore the communication feasibility depends on several variables, such as the carrier communication frequency, point to point distance, the chemical composition of water, the topology of the seafloor, the temperature of the water, the depth of sensors placement and the spreading pattern.

Underwater acoustic sensors networks (UWASN) have several challenges that should be considered for a successful implementation. The first one is related to the lossy nature of the channel. Although acoustic signals can propagate well beneath the water, they suffer an important attenuation mainly produced by absorption; and this attenuation is proportional to the distance and the frequency of the signal. There are some other factors contributing to the attenuation, like the scattering and reverberation in the surface, because wind moves the reflection point in the surface. There is also reflection in vessels that may introduce Doppler effect in the signal. Moreover, the geometric spreading of the signal produces path loss in the transmission; regardless we are using spherical (that is common in deep water) and cylindrical spreading (that is more common in shallow water).

The second challenge refers to the nature of the acoustic signal used for transmitting the information. There are several man-made noises present in the ocean, and specially in the surf zone where it is usual to have a higher density, vessel traffic, sport activities and city noise. Another source of noise is provided by the environment itself; i.e., waves, winds, rains, animals and even seismic noise that may interfere with data transmission in the acoustic sensor network.

The third challenge is related to the multi-path characterization of the signals. Different signals may interfere between each other by means of the inter-symbol interference. The vertical links usually have little dispersion, so it is not common to have multi-path interference; however, in horizontal channels the spread may be significant and therefore different messages may interfere among them.

The fourth challenge to be addressed is the energy consumption. Underwater sensor networks are battery-powered, which also represents a mayor concern during its operation. The energy consumption during standby and data reception is low in commercial modems, but in data transmission the consumption depends on the distance that the signal should travel. The relationship between transmission power vs distance follows a quadratic function. One way to save the energy in these networks is by transmitting at a higher baud rates for short times, limiting the operating range. On the other hand, transmissions through long distances require high power and lower baud-rates; thus, high data volumes result incompatible with large distances under the power consumption point of view. Considering that in sensor networks the data volume is usually low, and assuming average ocean parameters, the speed of sound in the water is closed to 1.5 $\mathrm{km} / \mathrm{s}$, the carrier frequency is between $20 \mathrm{kHz}$ and $70 \mathrm{kHz}$ for distances larger than $1 \mathrm{~km}$. The operating range to maintain sustainable communications at reasonable baud rate, and with low corrupted data rates, is limited to $2 \mathrm{~km}$ point-to-point. The variance in the transmission times introduces uncertainty, therefore the round trip time for a message is usually pessimistically evaluated degrading the performance of the system. 
There is a transversal component in these sensor networks that can be used to help address most of these challenges. This component is the medium access control (MAC) protocol used by the UWASN. Considering the needs and the challenges to implement underwater acoustic sensors networks, and the limitations that the current approaches for implementing MAC protocol for this type of networks, this paper proposes an extended medium access control protocol based on Time Division Multiple Access (TDMA), but specifically for UWASN. The transmission mechanism is inspired in the self-organized TDMA [3] and the ad-hoc self-organized TDMA [4]. However, its operation is simpler than the previous ones, involving only two phases for the configuration and operation. For the slot selection process it uses a simple carrier sense multiple access/collision avoidance (CSMA/CA) mechanism.

The rest of the paper is organized in the following way. In Section 2 a short review of previous work is presented. In Section 3 the proposed MAC protocol is presented. In Section 4 the evaluation process of this proposal is presented and discussed. Finally, in Section 5 we presents the conclusions and future work.

\section{Related work}

This kind of networks has several aspects to consider, which are not yet defined, like the physical layer selection, link layer protocols, and network and transport layer protocols. The literature shows three main approaches to implement medium access control protocol (MAC): frequency division multiple-access (FDMA), carrier sense multiple access with collision avoidance (CSMA/CA) and time division multiple access (TDMA). The first one is not suitable for underwater acoustic transmission, due to the narrow bandwidth in underwater acoustic channels and the vulnerability of limited band systems to fading and multi-path. CSMA/CA has been proposed in previous work, but it is limited by the hidden station problem and the high propagation and variable delay. Long time periods are needed in each message transmission to guarantee that there has been no collisions degrading the overall performance. Finally, the TDMA schemes have problems with the synchronization, latency and unused bandwidth. However, for real-time transmissions it is the only option that can provide bounded delays, although it is necessary to leave several slots empty. In this section we discuss previous work mainly related to the medium access mechanism and its appropriateness for being used in underwater acoustic sensors networks.

In [5] the authors proposed the Multiple Access Collision Avoidance (MACA) protocol based on the use of short messages request to send (RTS) and clear to send (CTS), followed by the sequence of messages DATA and ACK. This mechanism has been successfully used in the Seaweb project [6]. Before sending data, the node should reserve the channel by issuing a RTS, and after that it has to wait for the CTS answer. Nearby stations also listen the request and wait for the answer. In the case that a neighbor station does not listen the CTS answer, it means there is no interference in the receiver, and that the transmission may proceed solving in this way the exposed problem. Any station, other than the original RTS sender, on hearing CTS will defer its transmission. In case of collisions of RTS messages, the nodes use a binary backoff algorithm to solve the conflict. 
In [7], the authors extend the MACA protocol and named it MACA for wireless (MACAW). They propose a less aggressive backoff algorithm for the link layer introducing the sequence RTS-CTS-DATA-ACK. In lightly loaded scenarios this mechanism has less performance, but with high loads it has much better throughput and fairer allocation.

In [8] another MACA-based protocol is proposed. In this case, the Floor Acquisition Multiple Access protocol requires that every transmitting station should acquire the floor control (in a wireless channel), before sending any data packet. Both the sender and the receiver should perform the collision avoidance to guarantee the control of the channel.

In [9], the authors introduce the UW-MAC for acoustic underwater sensor networks. The MAC scheme is based on CDMA with a novel closed-loop distributed algorithm to set the optimal transmit power and code length to minimize the near-far effect. The algorithm compensates the multi-path effect by exploiting the time diversity in the underwater channel, thus achieving high channel reuse and low number of packet retransmissions. The protocol works both in deep and shallow water, and involving static or mobile nodes.

SO-TDMA [3] and ASO-TDMA [4] use a self organized TDMA scheme for the access control. However, these protocols were thought for VHF radio frequencies and they are used for the localization of vessels and ships in the ocean.

The UWSO-TDMA proposed in this paper is a simple mechanism that uses TDMA. In contrast to the other MAC protocols, this approach can be used for real-time communications, as it is possible to bound the transmission delay.

\section{Description of the UWSO-TDMA Protocol}

The proposed protocol is based on the predictability of TDMA, rather than on the probabilistic CSMA/CA approach. Time is divided in slots, where a message fully occupies one time slot. Like in SO-TDMA [3], UWSO-TDMA uses a structured access channel for slot assignment. The slots allocation is performed in a distributed way, where each station/node in the network chooses the best slot to transmit. This is a decentralized scheme where nodes are responsible for sharing the communication channel and the synchronization. In the case of the UWASN, GPS information is not available under the water, therefore the synchronization source is usually a sonobuoy anchored in the region that is in charge of synchronizing the clocks of the underwater nodes. The sonobuoy uses the UTC time for synchronizing the network.

Since acoustic signals are attenuated, not all the sensors listen to the sonobuoy synchronization slot. When a station is not within the sonobuoy transmission range, it is synchronized in a second phase by an already synchronized node. To do this, the frame is divided in $m$ sub-frames $(S F)$. Within the first sub-frame, $S F_{1}$, only the nodes listening to the sonobuoy can transmit. In $S F_{2}$, the nodes at two hops of the SB can transmit. Nodes in $S F_{2}$ are synchronized by nodes in $S F_{1}$. With this hierarchical distribution, nodes in $S F_{j}$ are synchronized by nodes in $S F_{j-1}$. As nodes in $S F_{i}$ with $i>1$ are not in direct contact with the SB, their messages have to be aggregated by nodes in previous $S F$. Figure 1 shows the frame structure. 


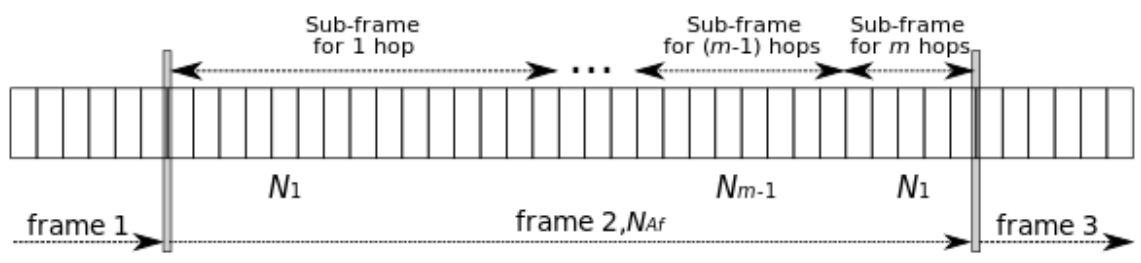

Fig. 1: Frame Structure (from [4])

The slot length is defined according to the data rate and message length. Based on these variables, the number of available slots in a frame are determined, $N_{A F}$. The number of slots in a sub-frame, $N_{k}$ is a function of the nodes within that hop. Finally, the number of hops or sub-frames are defined according to the expected physical deployment of the UWASN.

\subsection{Initialization}

Figure 2 presents the general flowchart for the this phase. For ease of understanding, details on the slot selection process are not represented. In this phase the nodes scan one frame to locate idle time slots and check the existence of neighbor nodes. The sonobuoy transmits in the first slot of the frame. In that slot, this node sends the current time so nodes listening to this first slot can determine their distance to the sonobuoy. However, some nodes probably are out of the transmission radio of the sonobuoy; for these cases, the synchronization is made as explained before, through nodes in different sub-frames, $S F_{i}$.

Figure 3 presents an example with a possible distribution of nodes in a UWASN. The sonobuoy is noted as SB and it is the center of the red circle that marks the communication range of the SB. As can be seen, two nodes may be in direct contact with the $\mathrm{SB}$, but do not between them (e.g., nodes 1 and 2). Weather they are in direct contact or not, these nodes should select different slots within the first sub-frame to avoid both collisions and receiver collisions in the SB. In the next hop area, dotted red circle, there are 3 more nodes. Like in the previous case, even if they are at similar distance from the $\mathrm{SB}$, that is within the second hop, they have different data paths. A message starting in node 5, may need to go through nodes 4 and 2 before reaching the SB. Instead, node 3 is within range of node 2, so messages beginning in node 3 pass through node 2 before reaching the SB. This example shows that even if nodes 3, 4 and 5 are within a distance of two communication radios from the SB, the amount of hops needed to reach such a node is different for each one.

During this phase, nodes build the network topology to determine the path towards the SB. Each node in the system selects a slot in the frame and informs its depth, distance to the SB (if it is within one hop) or the accumulated distance and the path towards it. In the example of Figure 3, node 4 informs the distance to the SB and the path through 


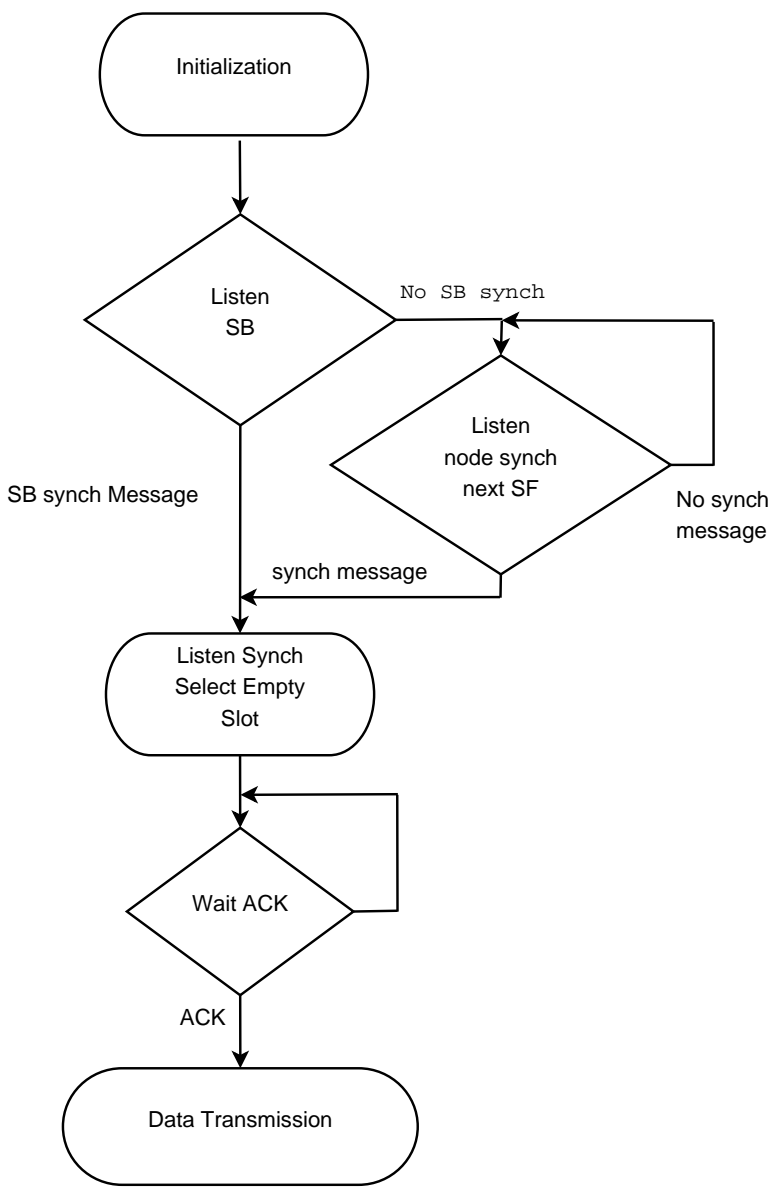

Fig. 2: Initialization Flowchart

node 2 . In that way, node 5 can synchronize its clock and determine its own distance to the SB and path.

The selection of the slot may require several steps to avoid collisions. To facilitate this process, UWSO-TDMA divides the frame in sub-frames like in [4]. The amount of available slots in a sub-frame is a designer decision, based on the amount of expected nodes in each region of the network. The sub-frame should have at least the same amount of available slots as nodes within the area.

Slot Selection Process The slot selection process uses a collision avoidance algorithm. After listening to the synchronization node, for example the SB, each node scans the sub-frame for an available slot and selects one. In the following frame, the nodes transmit in the selected slot. After this, three things may happen:

- There is no collision at all. In the next frame, nodes in the previous sub-frame or the SB (if the node is in direct contact with it) will acknowledge the selection. 
- Two or more nodes within transmission range select the same slot. They will detect the collision and stop. Then, they follow a backoff algorithm to select another slot.

- Two or more nodes are not within transmission range, so they do not detect the collision in the previous nodes sub-frame. In this condition, the nodes will not receive the acknowledgment, therefore they assume that there has been a collision and follow a backoff algorithm to select a new slot.

During the backoff, the nodes select one slot randomly from the set of available ones in the sub-frame. As there is no master allocating the slots and to avoid a long selection process, sub-frames have more available slots than nodes in the area. The probability of two nodes selecting the same slot is equal to $1 / n^{2}$, where $n$ is the amount of idle slots in the sub-frame at the moment of the selection. As there is at least one available slot after all nodes have selected their own slot, the worst case is when two nodes compete for three slots. In this situation, the probability of collision is 0.11 . If two slots are left idle, the probability decays to 0.06 and if three slots are left empty to 0.04 .

The SB is in charge of reinitializing the network periodically. This process is repeated to shuffle the slot assignments and to facilitate the incorporation of new nodes. As frames are synchronized with the UTC and begins with the minute, the period is a designer decision based on the conditions of the UWASN. For example, in networks deployed in places with strong currents is probable that nodes even if anchored to the bottom are moved. The reinitialization procedure facilitates also the redefinition of the nodes in charged of aggregating the information from lower SF nodes.

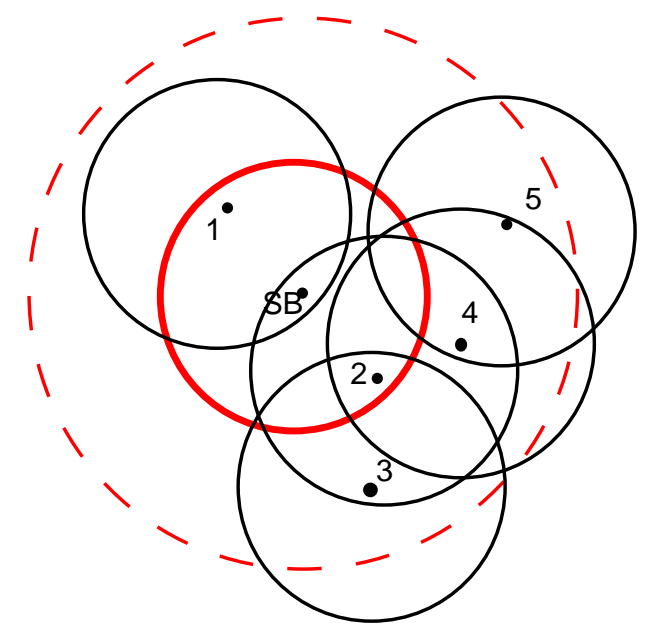

Fig. 3: Case 1: Deployment and transmission ranges

\subsection{Data Transmission}

Figure 4 shows the flowchart for the data transmission phase. In this phase, nodes transmit their data in the selected slot together with the distance to the SB and depth. As all nodes within range receive this information, they are able to keep an updated version 
of the network topology. As underwater transmission is prone to have data corruption, each message should be acknowledged after being received without corruption, by the synchronizing nodes in the previous sub-frames. If a message is not acknowledged, the sender retransmits it in the next frame. In the case a new message is generated, the information is aggregated or the old message is discarded, but this situation is flagged, so upstream nodes may notice the missing one. At the beginning of every frame, nodes wait for the synchronization message. If this message is not received with the acknowledgments of previous messages, the nodes in the sub-frame should assume that the synchronization node is out of work, and the backup synchronization node should be used if available. If there is not a backup, the node goes into the initialization phase.

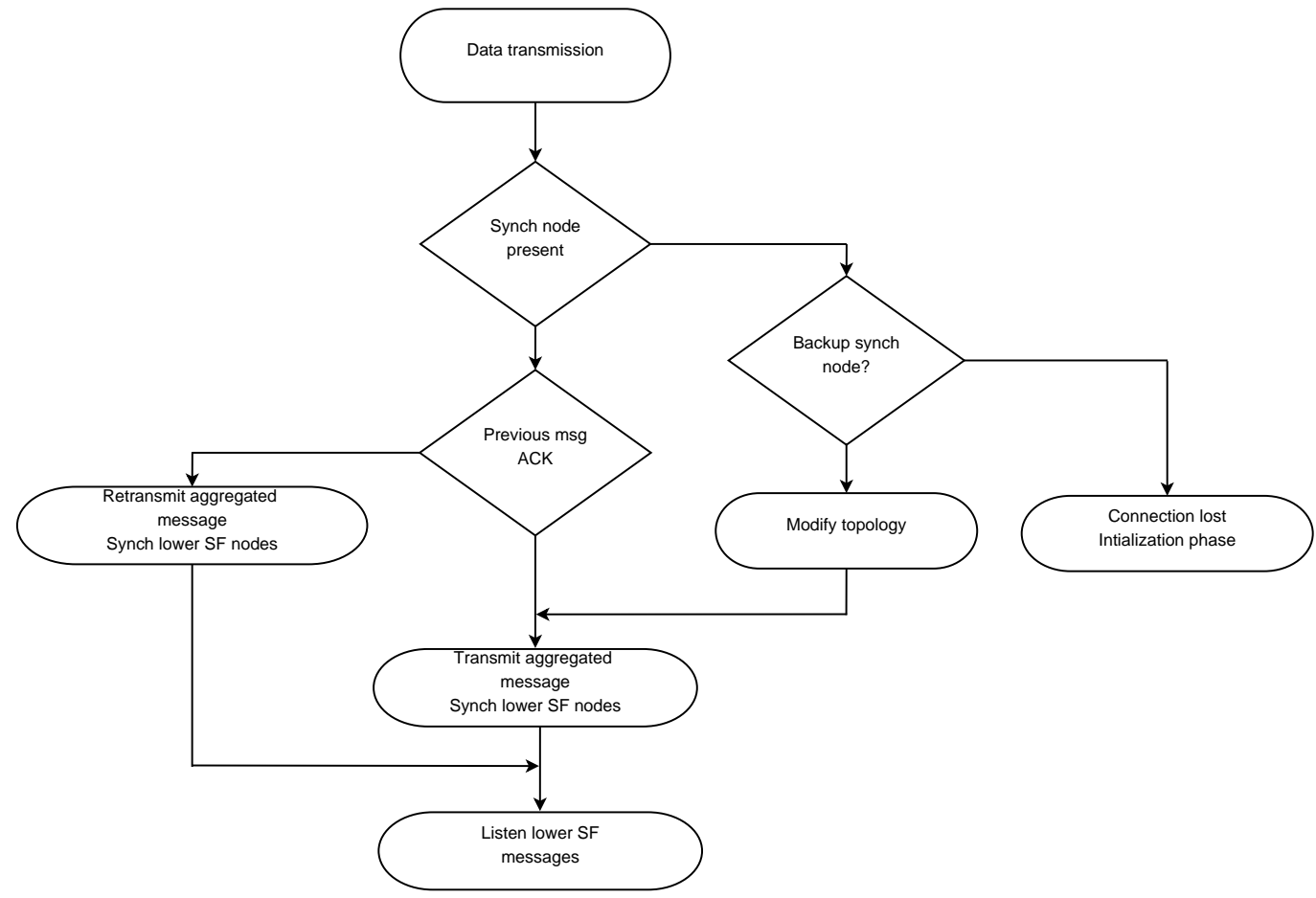

Fig. 4: Data transmission flowchart

In the example presented in Figure 3, the node 5 is the last node. Its messages have to go through nodes 4 and 2 to reach the SB. In this case, node 4 has to aggregate the information of node 5 in its own message and in the same way node 2 aggregates the information from nodes 4 and 5 in its own message. Figure 5 presents a case where more nodes are incorporated. In this case, the path from 6 to the SB is through node 1. However, as can be seen, the node 7 listens to nodes 5 and 6 . Node 5 is in the third sub-frame (SF), but node 6 is in the second one. The aggregation process is made only by nodes in the previous SF. When there is more than one node in the previous SF that listens to the messages from lower SF nodes, only one node aggregates and forwards the information to the next SF. The other nodes keep a copy of the information as backup, 
in case there is a failure in the transmission. Even if the path with the shortest delay is preferable, this scheme suppose the use of only one node as gateway to the next SF. The idea is that the first listened node in the SF is in charge of aggregating the information. As the slot allocation procedure is repeated in each periodic initialization phase, the nodes doing the aggregation are also changed.

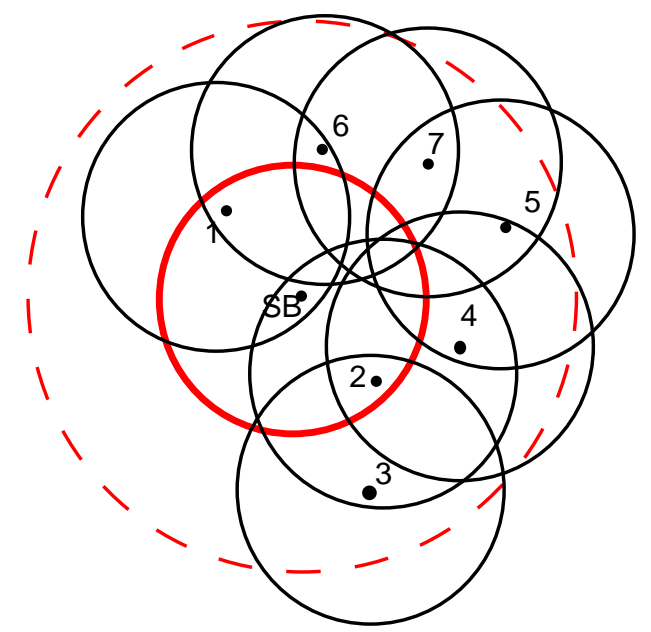

Fig. 5: Case 2: Deployment and transmission ranges

Figure 6 presents the topology of the UWASN represented in Figure 5. The dotted lines represent the different sub-frames used in the transmission of messages. As can be seen, if node 6 disappears, the network will be reconfigured and node 7 will be in a new sub-frame behind node 5 . In the same way, if node 4 is turned off, node 5 will send its messages through node 7 .

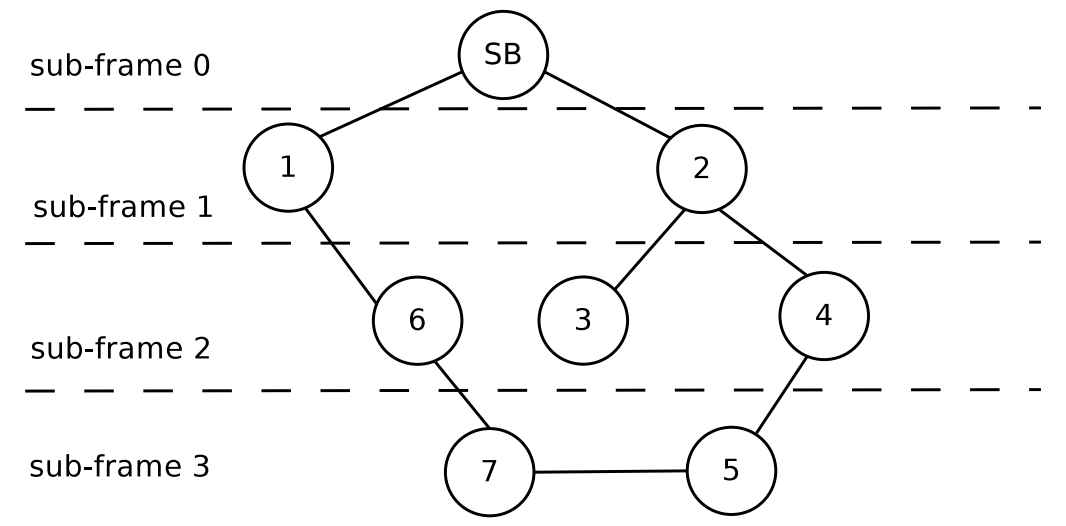

Fig. 6: Topology of the network in case 2 


\subsection{Delay Management}

The propagation speed of underwater acoustic signals is quite slow in relation to the amount of information that can be transferred through the air. In the case of encoding messages at $9600 \mathrm{bits} / \mathrm{s}$, in the time required for the signal to cover $1.5 \mathrm{~km}$ (it is one second), the transmitter should send 1200 bytes. Usually, messages are much shorter, so they will require only a few milliseconds. This fact introduces an important synchronization problem between sources and destinations if acknowledgments are required for each message. The UWSO-TDMA solves this problem using slot identification and time stamps in every message. In this way, even if the round trip of a message requires an important delay, it can be handled.

\section{Evaluation Process}

In this section we evaluate the performance of the MAC proposal using the example introduced in the previous section. We compare the general behavior of our proposal with the one of the MACA-U protocol [10]. For this evaluation process we assume that each node has an acoustic range equal to 500 meters, the carrier frequency is set to 40Khz [11] and the transmission data rate to 9600 bits/s. Each slot takes $167 \mathrm{~ms}$ for 160 bytes. Figure 7 shows the frame structure after the initialization phase for the network topology represented in Figure 6.

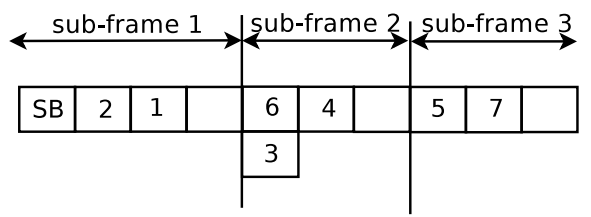

Fig. 7: Slot allocation in the example frame

As can be seen there are 10 slots used with 3 empty ones. The empty slots are used to reduce the probability of collisions in the selection slot process. The complete frame is transmitted in 1.67 seconds. In ASO-TDMA and SO-TDMA, the frame has 2650 slots and takes a whole minute. Nodes in the network have access to a GPS or UTC time, so they can synchronize their clocks and capture the beginning of the frames in the first instant of every minute. In the case of UWASN, only the sonobuoy has access to the UTC. Frames usually have less slots due the number of nodes are known.

Frames can start with different periodicity, for example every 10 seconds. In this case, the power demand (i.e., energy consumption) of the communication system in the node will be less than $20 \%$. After the initialization phase, a message from the farthest node (in our case, node 7), requires three frames to reach the $S B$ or 30 seconds. The ACK message is issued in three steps. First, node 6 acknowledges the reception of the message to node 7 and in the next frame, then node 1 acknowledges node 6 in the following frame, and finally the $S B$ acknowledges the reception of the message from node 1 . In steady state it is possible to send a message every 10 seconds, receiving the acknowledgment in the following 10 seconds, and the general utilization ratio of the network is below $20 \%$. 
In case of MACA-U protocol, the nodes can transmit whenever they have information available and they can lock the access to the acoustic channel, by issuing a RTS-CTS message with the next hop in the network. In this case, a timer is set with both, the time needed to send the message and the maximum delay for the message to reach the next node. Considering that the underwater acoustic signal propagates at 1.5 $\mathrm{km} / \mathrm{s}$, the time needed to reach the farthest possible node is equal to 0.333 seconds; therefore, the maximum round trip $\tau_{\max }$ is equal to 0.667 seconds. The same time slot is kept for comparison purposes 0.167 seconds. In case node 7 has a message to send, it will send an RTS message and wait for $\tau_{\max }+t_{\text {slot }}=0.834$ seconds. If in this time this node receives a CTS message, then it gains access and transmits its message. So, the total time needed by node 7 to send its message to the sonobuoy is 3 seconds without collisions. However, node 7 may be delayed by collisions with nodes 5 and 6 . In this case, and assuming only one collision with each one, the message from node 7 will only be transmitted to node 6 after 3 seconds. Node 6 can be delayed by collisions with node 1 and 7 . Therefore, considering again only one collision with each one, the message needs another 3 seconds to reach node 1 . In the last hop, node 1 may have collisions with nodes 6,2 and the sonobuoy, and like in the previous cases, the message will be delayed for another 4 seconds. The end-to-end transmission time for a message originating in node 7 is the sum of the partial delays, i.e., 10 seconds.

This simple example shows that the complexity of a collision avoidance algorithm for transmission of information in networks with important delays may compromise its performance severely. Another important aspect is that the delay is not deterministic, showing great variations according to the network load. Instead, once the slot selection process is done, the TDMA approach guarantees a constant latency and transmission delay, making the message propagation predictable.

\section{Conclusions and Future Work}

In this paper we have proposed the UWSO-TDMA MAC protocol for underwater acoustic sensor networks. The mechanism is based on a TDMA protocol, but without including a master node or a fixed schedule for the nodes. In fact, each node selects the slot in which it will transmit in a distributed way. Time synchronization is kept through a sonobuoy that picks up the time from GPS or Galileo system. Nodes that are not within the sonobuoy transmission range, synchronize their clocks through retransmitting nodes. As the protocol is based on TDMA, transmission delay and latency are bounded, and the network can be used for transmitting messages with real-time constraints.

Although this proposal is still being evaluated, the preliminary results are encouraging. Addressing the data transmission in UWASN opens new opportunities for studying several environments that have been difficult to address for researchers. As part of the future work we intend to evaluate the performance of the proposed protocol in a real-world scenario: throughput, latency, delay against other protocols like MACA or MACAW. Depending on the obtained results we will determine specific opportunities and restrictions to use this proposal. 


\section{Acknowledgments}

This work was partially supported by the European Community through the project Community Networks Testbed for the Future Internet (CONFINE): FP7-288535, and also by Spanish goverment under contract TIN2013-47245-C2-1-R.

\section{References}

1. UNESCO: Facts and figures on marine biodiversity

2. Xu, G., Shen, W., Wang, X.: Applications of wireless sensor networks in marine environment monitoring: A survey. Sensors 14(9) 16932-16954

3. ITU: M.1371 : Technical characteristics for an automatic identification system using timedivision multiple access in the vhf maritime mobile band

4. Yun, C., Lim, Y.k.: Aso-tdma: ad-hoc self-organizing tdma protocol for shipborne ad-hoc networks. EURASIP Journal on Wireless Communications and Networking 2012(1) (2012)

5. Karn, P.: Maca - a new channel access method for packet radio. In: Proceedings of the 9th Computer Networking Conference ARRL/CRRL Amateur Radio. (September 1990) 134140

6. Rice, J., Creber, B., Fletcher, C., Baxley, P., Rogers, K., McDonald, K., Rees, D., Wolf, M., Merriam, S., Mehio, R., Proakis, J., Scussel, K., Porta, D., Baker, J., Hardiman, J., Green, D.: Evolution of seaweb underwater acoustic networking. In: OCEANS 2000 MTS/IEEE Conference and Exhibition. Volume 3. (2000) 2007-2017 vol.3

7. Bharghavan, V., Demers, A., Shenker, S., Zhang, L.: Macaw: A media access protocol for wireless lan's. SIGCOMM Comput. Commun. Rev. 24(4) (October 1994) 212-225

8. Fullmer, C.L., Garcia-Luna-Aceves, J.J.: Floor acquisition multiple access (fama) for packetradio networks. SIGCOMM Comput. Commun. Rev. 25(4) (October 1995) 262-273

9. Pompili, D., Melodia, T., Akyildiz, I.: A cdma-based medium access control for underwater acoustic sensor networks. Wireless Communications, IEEE Transactions on 8(4) (April 2009) 1899-1909

10. Ng, H.H., Soh, W.S., Motani, M.: Maca-u: A media access protocol for underwater acoustic networks. In: Global Telecommunications Conference, 2008. IEEE GLOBECOM 2008. IEEE. (Nov 2008) 1-5

11. EvoLogic: S2cm modem series 